\title{
Radio Galaxies: Physics and Surveys
}

\section{R.A. Laing*}

ESO, Karl-Schwarzschild-Straße 2, 85748 Garching-bei-München, Germany

E-mail: rlaing@eso.org

Two aspects of radio-source physics in which there are synergies between deep, single-object observations and large surveys are reviewed. The first is the deceleration of initially relativistic jets by entrainment and its relevance to the Fanaroff-Riley classification of radio galaxies. Detailed models of jet velocity fields have been derived for a small number of bright sources. Surveys will complement these studies by allowing us to determine the dependence of the deceleration process on jet energy flux and environment. The second example concerns magnetic fields in the thermal plasma that surrounds radio galaxies. Surveys of significant numbers of radio galaxies will allow us to constrain the location of this magnetized plasma and to characterize the field geometry as ordered or tangled.

EXTRA-RADSUR2015 (*)

20-23 October 2015

Bologna, Italy

(*) This conference has been organized with the support of the Ministry of Foreign Affairs and International Cooperation, Directorate General for the Country Promotion (Bilateral Grant Agreement ZA14GR02 - Mapping the Universe on the Pathway to SKA)

${ }^{*}$ Speaker. 


\section{Introduction}

Jets - fast, highly-collimated, bipolar outflows - are inextricably linked to the processes of accretion and collapse onto compact astrophysical objects. They are observed in Young Stellar Objects, micro-quasars, pulsar wind nebulae, $\gamma$-ray burst sources, tidal disruption events and, most spectacularly, in the objects that are the subject of this paper, radio galaxies. Not only can these jets be the primary channel of energy loss from accreting supermassive black holes, but they also have a major impact on their surroundings (thereby significantly affecting galaxy evolution), and act as accelerators of the most energetic photons (and perhaps hadrons) we observe. The quantitative study of relativistic jets is notoriously difficult, because they emit primarily by the broad-band synchrotron and inverse Compton processes which offer few diagnostics of physical parameters. Nevertheless, progress in understanding jet physics on kiloparsec scales has recently come from detailed multi-wavelength observations of individual objects, as I outline below. In pondering what someone who therefore writes several papers on one pair of radio jets was doing at a meeting about radio surveys, I came to the conclusion that the question I could most usefully try to answer was "What can we learn from the combination of deep, high-resolution, single-object observations and shallower surveys?" As examples, I have chosen two aspects of radio-galaxy physics for which this question can sensibly be be asked: velocities in jets and magnetic fields in the surrounding intergalactic medium (IGM). These are addressed in Sections 2 and 3 respectively.

\section{Jet velocities and the Fanaroff-Riley Classification}

\subsection{Fanaroff-Riley classes}

The morphological classification of extended radio structures defined by Fanaroff \& Riley [4] has proved to be one of the most durable in the subject. FR I and FR II sources are centre- and edge-brightened, respectively (Fig. 1 shows examples). The key physical difference appears to be that the jets in FR I sources decelerate from relativistic to sub-relativistic speeds on kiloparsec scales, but that those in FR II's remain relativistic until they terminate in hot-spots at the ends of their radio lobes. The essential result of [4] is that the FR I sources have lower radio luminosities, the division between the classes ${ }^{1}$ being at $P_{1.4 \mathrm{GHz}} \approx 10^{25} \mathrm{~W} \mathrm{~Hz}^{-1}$.

It now seems likely that the FR division is determined primarily by the energy flux of the jet and the external environment through which it propagates, rather than being connected directly to some other property of the central engine such as accretion rate or black hole spin [2]. The dependence on environment is not quantitatively understood, however. One might expect the hot IGM to play a key role in jet deceleration by external entrainment, but mass input from stars (which in turn depends on stellar density and type) could also be important.

Ledlow \& Owen [13] found a strong dependence of the division on the optical luminosity of the host galaxy $\left(\propto P_{\text {opt }}^{2}\right)$, to the extent that FR I and II sources were separated cleanly in a plot of $P_{\mathrm{opt}}$ against $P_{1.4 \mathrm{GHz}}$, although their sample was not homogeneous. For a much larger sample selected uniformly by cross-matching radio sources with morphological classifications from NVSS and FIRST with galaxies in SDSS [2], there is much more overlap between the populations. In

\footnotetext{
${ }^{1} H_{0}=70 \mathrm{~km} \mathrm{~s}^{-1} \mathrm{Mpc}^{-1}$
} 


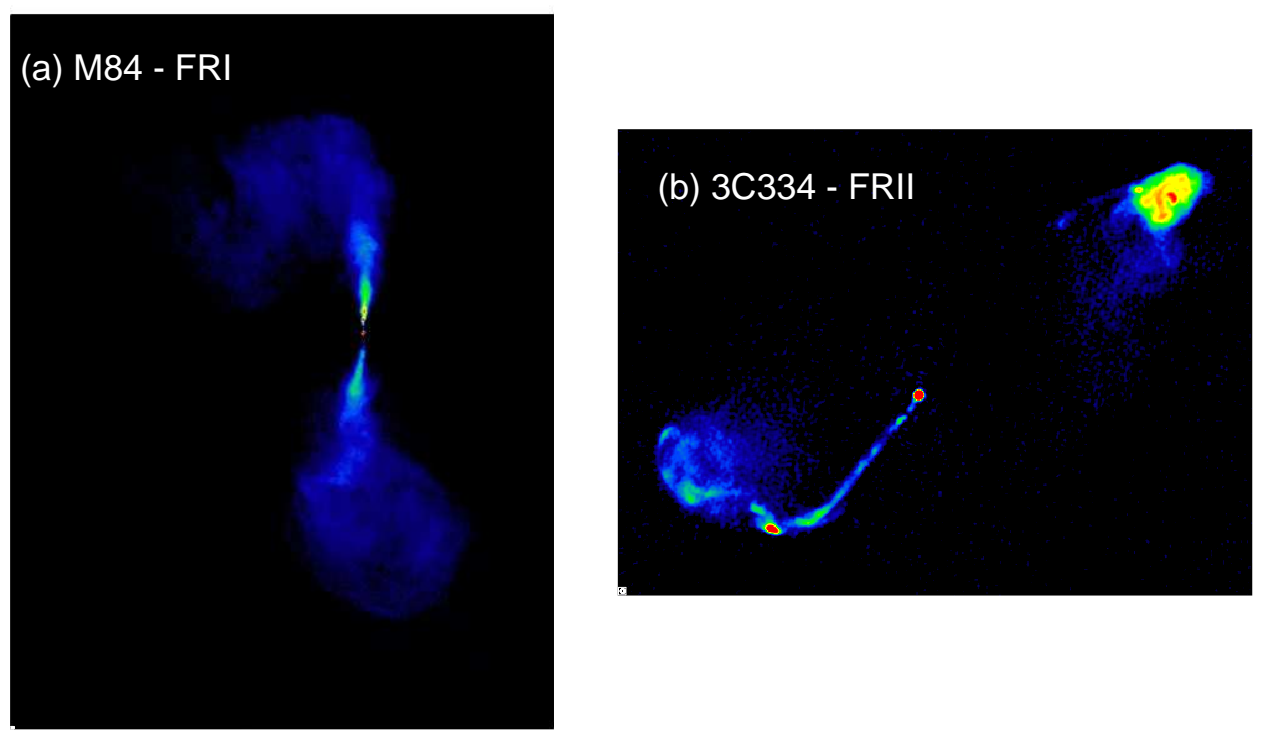

Figure 1: Examples of Fanaroff-Riley classes. (a) M 84 (FR I); (b) 3C 334 (FR II), both observed with the VLA at $4.9 \mathrm{GHz}[3,11]$.

the overlap region probed by the latter study, FR I and II host galaxies appear very similar in stellar age and velocity dispersion, differing only in concentration index. The restriction to sources whose morphology can be classified reliably using data from FIRST and/or NVSS still introduces significant selection effects, however. In order to remove this restriction, an arcsecond-resolution imaging survey of a large number of nearby radio galaxies will be needed.

\subsection{Flow speeds in FR I jets}

There is good evidence both from proper-motion measurements [14, and references therein] and from side-to-side asymmetries that the jets of FR I radio galaxies are relativistic (with bulk Lorentz factors $\Gamma \gtrsim 2$ ) out to distances of a few kpc from the AGN. We have developed a modelling method which has enabled us for the first time to estimate the variations of velocity, magnetic-field structure and proper emissivity of relativistic FR I jets in three dimensions as well as their intrinsic geometries and orientations [8, and references therein]. We assume that the jets are intrinsically symmetrical and relativistic, so apparent differences between them (in both brightness and linear polarization) result from special relativistic aberration. We take parametrized models of geometry, velocity, magnetic-field ordering and proper emissivity, calculate the emission in Stokes $I, Q$ and $U$ by numerical integration, accounting for relativistic aberration and anisotropic rest-frame emission, convolve to the resolution of our VLA images and evaluate $\chi^{2}$ between model and data. We then optimize the model parameters to minimize $\chi^{2}$. An example fit is shown in Fig. 2. We find $\Gamma \approx 2$ where the jets first brighten appreciably. All of the modelled jets expand rapidly and decelerate abruptly (Fig. 3), thereafter either maintaining a much slower, constant (but still mildly relativistic) speed or slowing less rapidly. The jets also develop transverse velocity gradients, with edges typically $30 \%$ slower than the centres: this is strong evidence in favour of external entrainment as the cause of the deceleration. The resulting picture of the way in which FR I jets flare and decelerate is sketched in Fig. 4(a). The jets start to recollimate almost exactly where rapid 

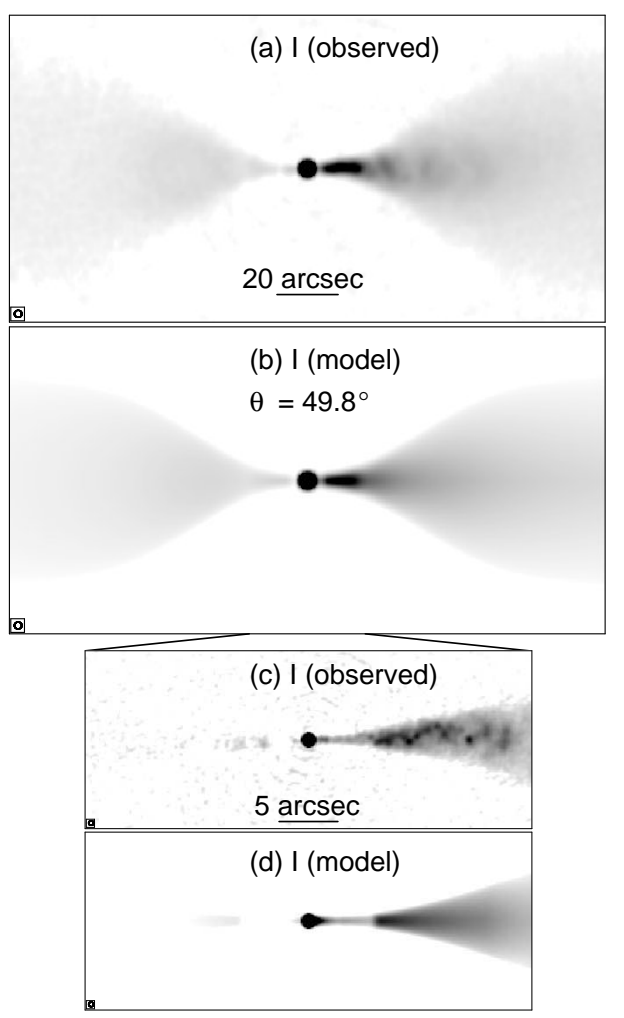
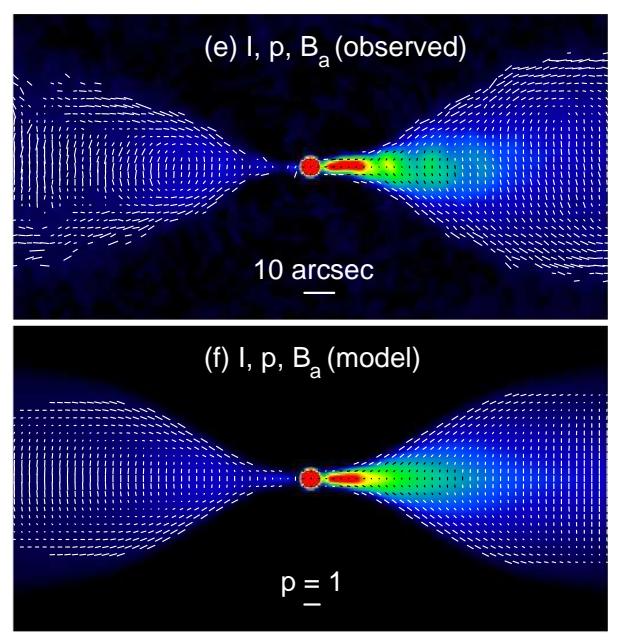

Figure 2: Observations and models of the nearby radio galaxy NGC 315 [8]. (a) observed and (b) model total intensity at 2.35-arcsec resolution. (c) and (d) as (a) and (b), but for the inner jets at 0.6-arcsec resolution. (e) and (f) Vectors with magnitudes proportional to the degree of polarization, $p$, and directions along the apparent magnetic field superposed on a false-colour plot of total intensity. (e) observations, (f) model.

deceleration ends (presumably when there is no longer much external material to be entrained), as illustrated in Fig. 4(b).

We have so far managed to estimate velocity fields for only 10 FR I radio jets, all of similar type and restricted to a limited range of size and radio luminosity [8]. We would like to know how the flaring and deceleration scales depend on jet power, galaxy (or black hole) mass and environment - particularly the density and pressure profiles of the surrounding hot plasma. One obvious role for surveys is to find more targets for detailed follow-up. In addition, although full modelling requires high sensitivity and angular resolution, profiles of the one-dimensional jet/counter-jet ratio $I_{\mathrm{j}} / I_{\mathrm{cj}}$ (which are much easier to measure) can be calibrated against the models and used to determine approximate inclinations (from the ratio close to the AGN) and velocity profiles (from the decrease in $I_{\mathrm{j}} / I_{\mathrm{cj}}$ with distance). Fig. 5 shows what could be achieved some years ago for the (small) B2 sample [12]. It plots the distance from the AGN at which the jet and counter-jet intensities become roughly equal (a good proxy for the deceleration scale) against total radio luminosity and the dependence is already apparent. Imaging surveys with sub-arcsecond resolution should provide much larger samples, with different types of source and environment.

The deceleration process for radio galaxies of very low luminosity $\left(\lesssim 10^{22} \mathrm{~W} \mathrm{~Hz}^{-1}\right.$ at $\left.1.4 \mathrm{GHz}\right)$ is poorly constrained, as there are inevitably very few examples near enough to be in the bright- 


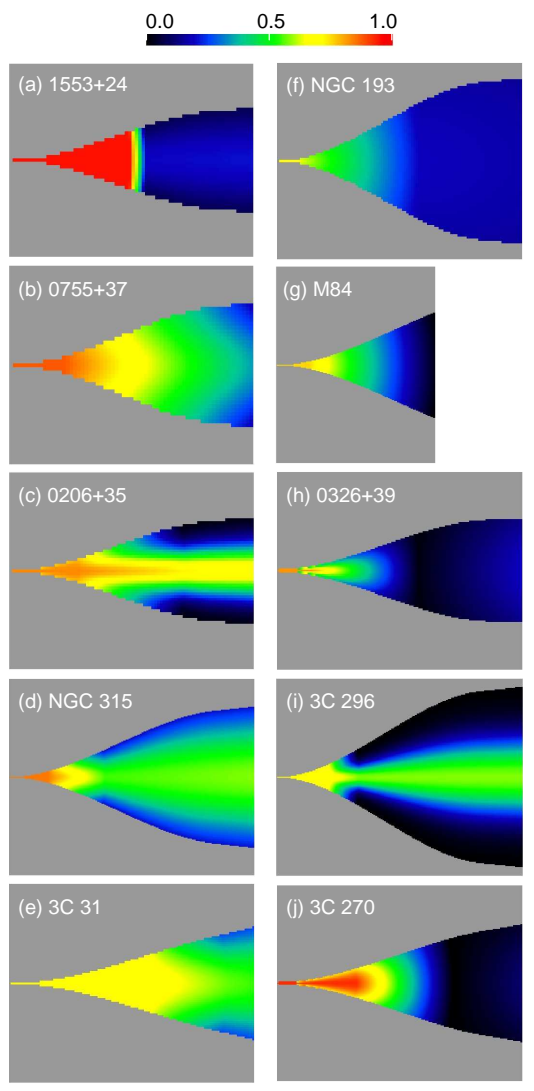

Figure 3: Model velocity fields for ten FR I jets from [8], in units of $c$.
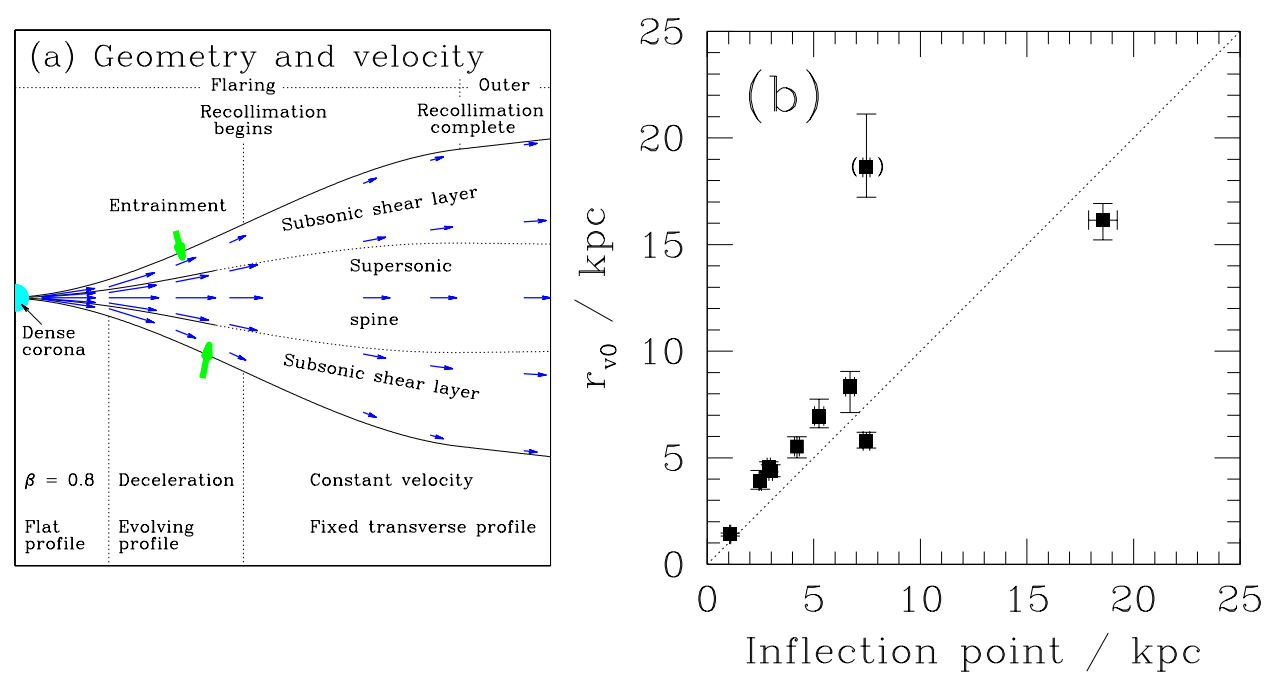

Figure 4: (a) A sketch of the evolution of the velocity field in a typical FR I jet and its relation to collimation. (b) A plot of the distance from the AGN at which rapid deceleration ends, $r_{v 0}$, against the distance at which recollimation begins, marked on panel (a). 
source catalogues like 3C and B2. The least luminous source whose jet velocity field has been modelled in detail, M $84\left(P_{1.4 \mathrm{GHz}}=10^{23.4} \mathrm{~W} \mathrm{~Hz}^{-1}\right)$, has a deceleration scale of $1.4 \mathrm{kpc}$ [8], while the weakest source in Fig. 5, B2 $1122+39$, has $P_{1.4 \mathrm{GHz}}=10^{22.0} \mathrm{~W} \mathrm{~Hz}^{-1}$ and a symmetrization distance of $0.7 \mathrm{kpc}$. The suspicion must be that sources in this power range still have initially relativistic jets, but decelerate over much shorter distances than their more luminous counterparts (see also [1]). A survey of large numbers of very low-luminosity radio galaxies with sub-kpc resolution could test this idea.

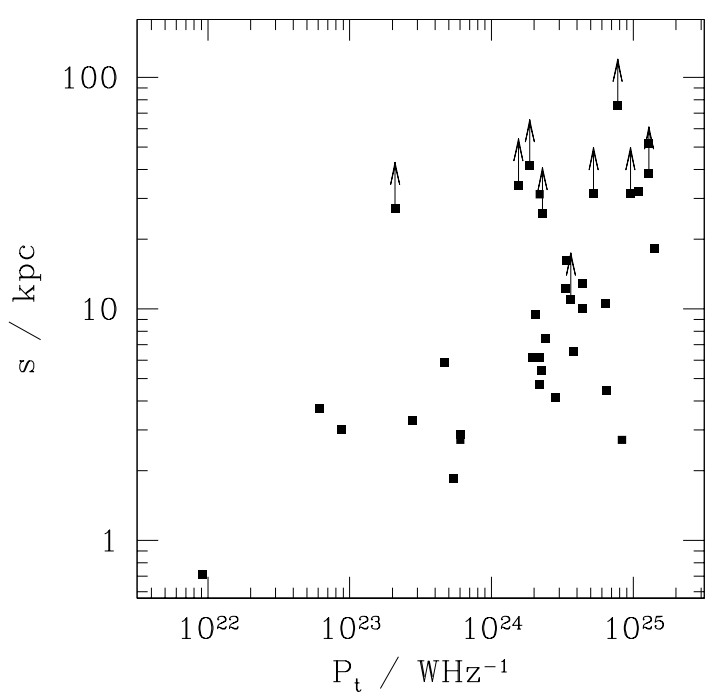

Figure 5: A plot of the distance at which jets become approximately symmetrical, $s$, against radio luminosity $P_{\mathrm{t}}$ for the B2 sample [12, corrected to $H_{0}=70 \mathrm{~km} \mathrm{~s}^{-1} \mathrm{Mpc}^{-1}$ ].

Another obvious role for surveys is to find unusual objects. A well-known historical example is the discovery of the spectacular jet in NGC 6251, which was identified as a giant radio galaxy in the $6 \mathrm{C}$ survey at $151 \mathrm{MHz}$ [15]. The reason that this remains a key object is that its jets are intermediate in expansion rate and sidedness ratio between the types found in FR I and FR II sources. An analysis of VLA archive data suggests that the central "spine" of the jet remains very fast $(\Gamma \gtrsim 5)$ even at large distances from the AGN [9] and deeper observations with the Jansky VLA have been taken to enable detailed modelling. This type of analysis will remain impossible for powerful FR II quasars such as 3C 334 (Fig. 1b) until the advent of SKA Phase 2 [7] and in the mean time a key role of surveys will be to search for more cases like NGC 6251.

\section{Magnetic fields in and around radio galaxies}

\subsection{Location of the magnetoionic medium}

Multi-frequency imaging enables the measurement of Faraday rotation - the rotation of the plane of polarization of linearly-polarized radiation propagating through a magnetized plasma. In most cases studied so far at high spatial resolution, the observed Faraday rotation is dominated by magnetized thermal plasma in front of (rather than mixed in with) the radio-emitting material. 


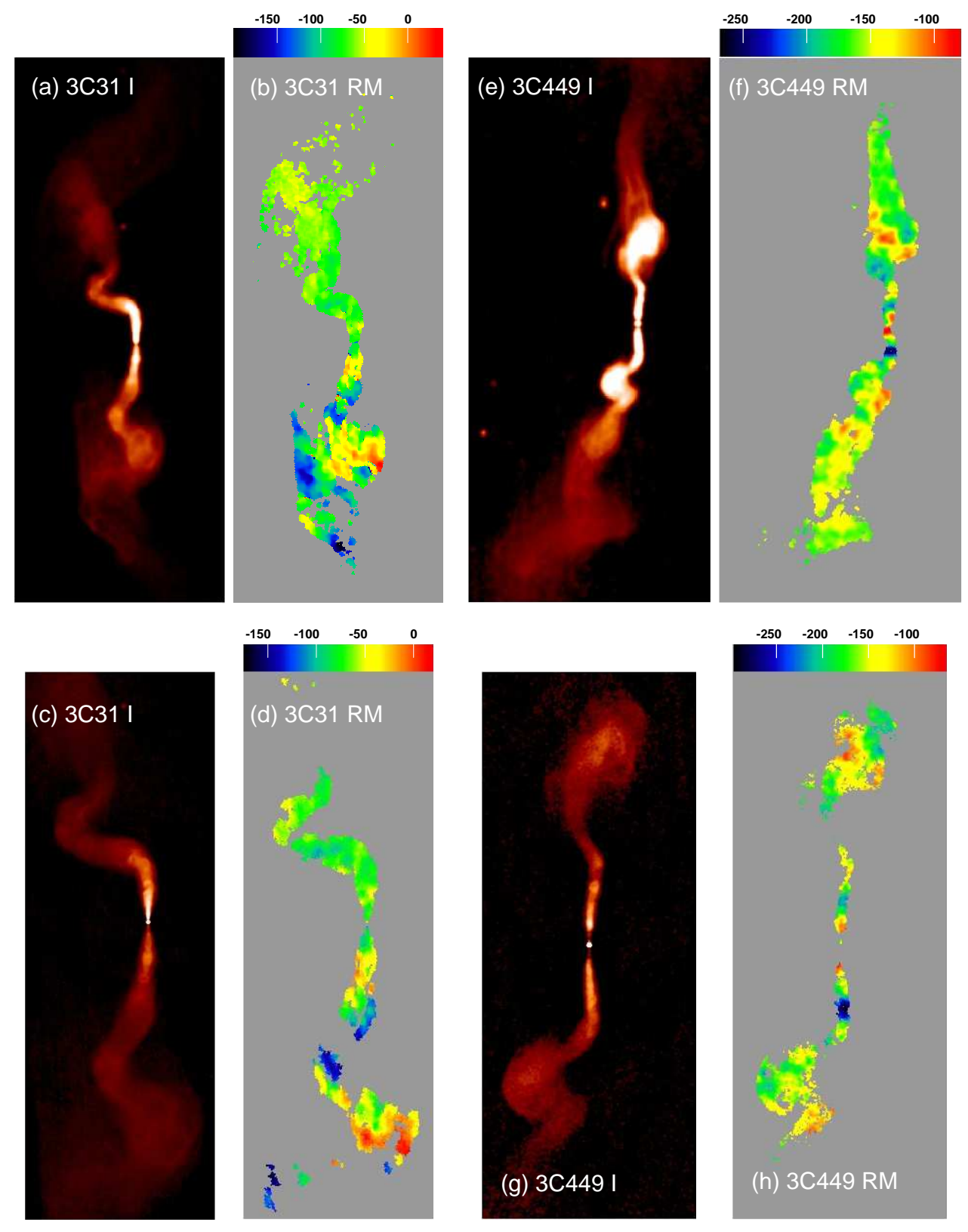

Figure 6: Total intensity and rotation-measure distributions for two FR I radio galaxies: 3C 31 (panels a - $\mathrm{d}$; [10]) and 3C 449 (panels e - h; [5]). All of the images have been rotated so that the approaching jet is uppermost. $3 \mathrm{C} 31$ has $\theta \approx 52^{\circ}$ and both the jets and the Faraday rotation fluctuations show marked asymmetries. $3 \mathrm{C} 449$ is close to the plane of the sky and is very symmetrical. The top and bottom rows show images at 5.5 and 1.5-arcsec resolution, respectively. 
We expect foreground Faraday rotation distributions to depend on source orientation. Emission from the near side of a radio galaxy will inevitably be seen though less thermal plasma and will therefore show less Faraday rotation. The jet-modelling technique described in Section 2 gives a quantitative estimate of the inclination which can be compared with observed asymmetries in the rms Faraday rotation measure, $\sigma_{\mathrm{RM}}$. The effect is illustrated in Fig. 6, which contrasts total intensity and RM distributions for two sources with very different inclinations. Fig. 7 shows results from [8]: significant asymmetries in the expected sense are observed whenever the inclination $\theta \lesssim 55^{\circ}$, but not for sources closer to the plane of the sky.

The RM asymmetry is qualitatively consistent with a picture in which the Faraday rotation is caused by magnetic fluctuations in a roughly spherical distribution of hot thermal plasma with a characteristic radius comparable with the source size (consistent with the observed X-ray emission). A more detailed analysis shows that this geometry is oversimplified in at least one case: 3C 31 [10]. The problem is that the change in $\sigma_{\mathrm{RM}}$ across the nucleus is much more abrupt than is expected if the plasma density is described by a beta model and the magnetic field strength scales with the density (Figs $6 \mathrm{~b}$ and d). Much better fits to the $\sigma_{\mathrm{RM}}$ profile can be obtained for a geometry in which the radio lobes have created large cavities in the IGM which are essentially devoid of thermal plasma, or if the field is primarily in a disk orthogonal to the jets [10]: in neither case is there much thermal material in front of the approaching jet.

As only a very few cases can yet be analysed at this level of detail, we do not know whether the type of $\sigma_{\mathrm{RM}}$ profile observed in $3 \mathrm{C} 31$ is typical or an accident of geometry. The role of surveys will be to provide a large sample of radio galaxies with Faraday rotation distributions resolved across the nucleus and at least a crude indicator of inclination derived from $I_{\mathrm{j}} / I_{\mathrm{cj}}$ where the jets first brighten, as in Section 2.2.

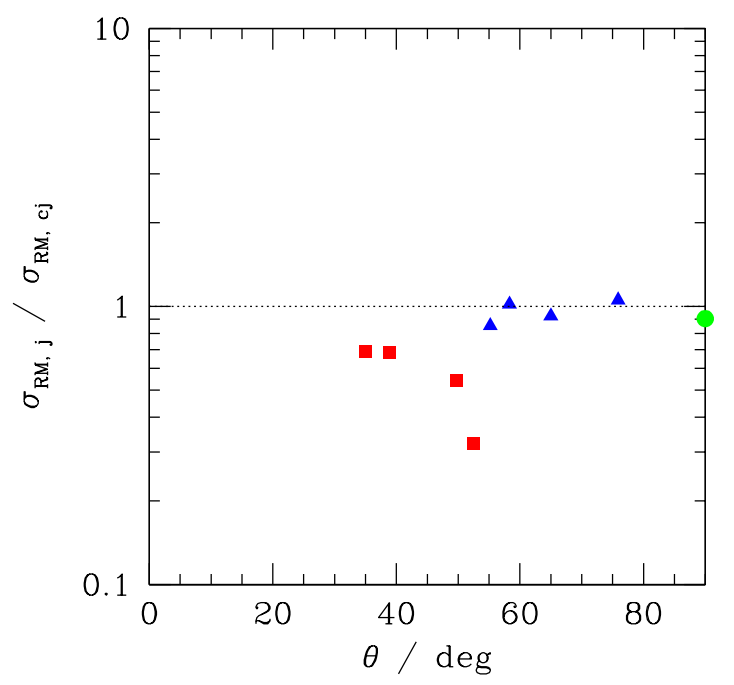

Figure 7: Ratios of rms Faraday rotation for the approaching and receding sides of 11 twin-jet sources plotted against angle to the line of sight, $\theta[8]$. 


\subsection{Field structure in the intergalactic medium}

Faraday rotation produced by the local foreground medium associated with radio galaxies can show one or both of two very different morphologies, as follows.

Disordered The RM distribution is patchy, as in 3C 31 and 449 (Fig. 6). For these systems, the field can be modelled as a Gaussian random variable with an approximately power-law power spectrum embedded in plasma with a smoothly varying density distribution. Key questions for this type of field structure relate to the form of the power spectrum. Is the slope typically flatter than Kolmogorov [5, 10]? What are the inner and outer scales? What is the field strength and how does it vary with plasma density? Is the field ever dynamically important?

Ordered A completely different type of field structure is needed to explain "RM bands" [6], with straight iso-RM contours orthogonal to the source axis, as seen in B2 0206+35 (Fig. 8). Here the field must be well-ordered, perhaps shaped by some magnetic draping process.

Estimation of the power spectrum in the disordered case requires well-sampled observations and deep X-ray images. Shallower surveys can, however, answer one important basic question: what is special about the radio galaxies that show ordered RM distributions? Do they always have lobes with sharp outer boundaries rather than diffuse plumes (compare Figs 6 and 8)? Is there anything unusual about the host galaxies? Surveys in Stokes $I Q U$ with linear resolution better than $\approx 500 \mathrm{pc}$ over a wide frequency range are required.
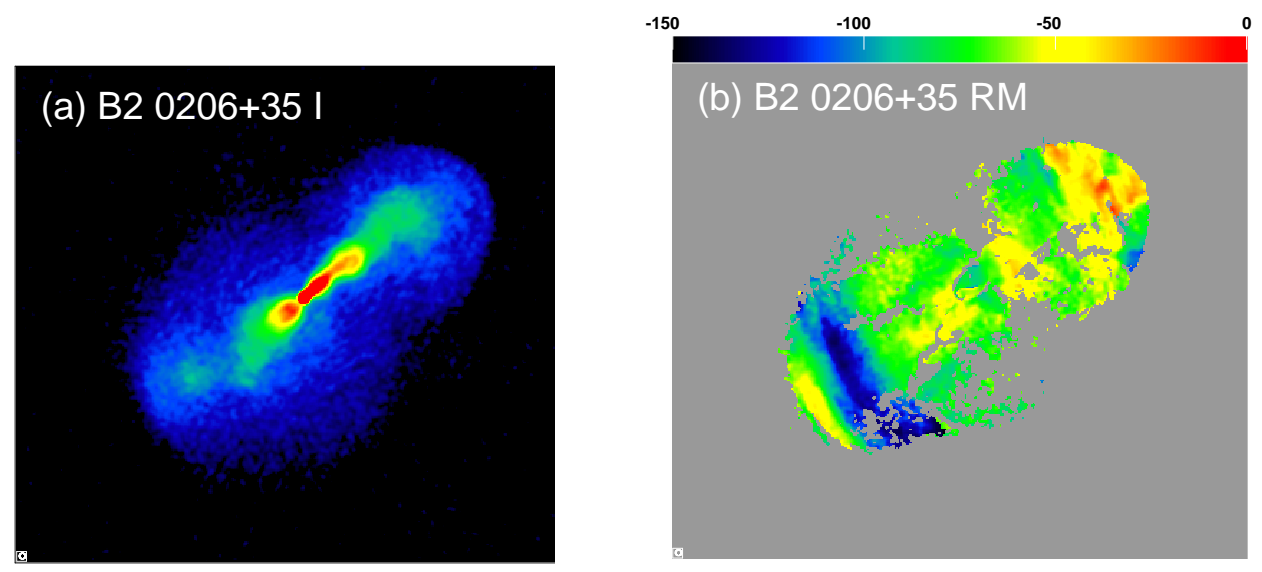

Figure 8: Total intensity and rotation measure images of B2 $0206+35$ at a resolution of $1.2 \operatorname{arcsec}$ FWHM. (a) Total intensity at $4.9 \mathrm{GHz}$ [11]. (b) Rotation measure, plotted over the range $-150-0 \mathrm{rad} \mathrm{m}^{-2}$ [6].

\section{Conclusions}

This paper has described two areas in which deep, detailed, high-resolution studies of single radio galaxies complement shallower surveys covering many objects. Good resolution and image quality are needed for the surveys, along with full polarization coverage over wide frequency ranges. 


\section{References}

[1] Baldi R.D., Capetti A., Giovannini G., Pilot study of the radio-emitting AGN population: the emerging new class of FR 0 radio-galaxies, 2015, A\&A 576 A38

[2] Best P.N., Radio source populations: Results from SDSS, 2009, AN 330184

[3] Bridle A.H., Hough D.H., Lonsdale C.J., Burns J.O., Laing R.A., Deep VLA imaging of twelve extended 3CR quasars, 1994, AJ 108766

[4] Fanaroff B.L., Riley J.M., The morphology of extragalactic radio sources of high and low luminosity, 1974, MNRAS 167 31P

[5] Guidetti D., Laing R.A., Murgia M., Govoni F., Parma P., Gregorini L., Feretti L., Structure of the magnetoionic medium around the Fanaroff-Riley Class I radio galaxy 3C 449, 2010, A\&A 51450

[6] Guidetti D., Laing R.A., Bridle A.H., Parma P., Gregorini L.Ordered magnetic fields around radio galaxies: evidence for interaction with the environment, 2011, MNRAS 4132525

[7] Laing R., Kinematics and Dynamics of kiloparsec-scale Jets in Radio Galaxies with SKA, 2015, in Advancing Astrophysics with the Square Kilometre Array, Pos(AASKA14), 107

[8] Laing R.A., Bridle A.H., Systematic properties of decelerating relativistic jets in low-luminosity radio galaxies, 2014, MNRAS 4373405

[9] Laing R.A., Bridle A.H., Kinematics of kiloparsec-scale jets, 2015, in Extragalactic Jets from Every Angle, IAU Symp. 313, 108

[10] Laing R.A., Bridle A.H., Parma P., Murgia M., Structures of the magnetoionic media around the Fanaroff-Riley Class I radio galaxies 3C31 and Hydra A, 2008, MNRAS 391521

[11] Laing R.A., Guidetti D., Bridle A.H., Parma P., Bondi M., Deep imaging of Fanaroff-Riley Class I radio galaxies with lobes, 2011, MNRAS 4172789

[12] Laing R.A., Parma P., de Ruiter H.R., Fanti R, Asymmetries in the jets of weak radio galaxies, 1999, MNRAS 306513

[13] Ledlow M.J., Owen F.N., 20 cm VLA Survey of Abell Clusters of Galaxies. VI. Radio/Optical Luminosity Functions, 1996, AJ 1129

[14] Meyer E.T. et al., A kiloparsec-scale internal shock collision in the jet of a nearby radio galaxy, 2015, Nature, $\mathbf{5 2 1} 495$

[15] Waggett P.C., Warner P.J., Baldwin J.E., NGC 6251, a very large radio galaxy with an exceptional jet, 1977, MNRAS 181465 